\title{
Development of High Temperature, Radiation Hard Detectors Based on Diamond
}

\author{
Alex Metcalfe ${ }^{\mathrm{a},}$, George R. Fern ${ }^{\mathrm{a}}$, Peter R. Hobson ${ }^{\mathrm{b}}$, Terry Ireland ${ }^{\mathrm{a}}$, Ali Salimian ${ }^{\mathrm{a}}$, Jack Silver $^{\mathrm{a}}$, David R. Smith ${ }^{\mathrm{b}}$, Gwenaelle \\ Lefeuvre $^{\mathrm{c}}$, Richard Saenger ${ }^{\mathrm{d}}$ \\ ${ }^{a}$ Wolfson Centre for Materials Processing, Brunel University London, Uxbridge, UB8 3PH, UK \\ ${ }^{b}$ Centre for Sensors $\mathcal{E}$ Instrumentation, College of Engineering, Design and Physical Sciences, Brunel University London, Uxbridge, UB8 3PH, UK \\ ${ }^{c}$ Micron Semiconductor Ltd, Lancing BN15 8 SJ, UK \\ ${ }^{d}$ Schlumberger Limited, 91240 Clamart, France
}

\begin{abstract}
Single crystal CVD diamond has many desirable properties compared to current, well developed, detector materials; exceptional radiation, chemical and physical hardness, chemical inertness, low Z (close to human tissue, good for dosimetry), wide bandgap and an intrinsic pathway to fast neutron detection through the ${ }^{12} \mathrm{C}(\mathrm{n}, \alpha){ }^{9} \mathrm{Be}$ reaction. However effective exploitation of these properties requires development of a suitable metallisation scheme to give stable contacts for high temperature applications. To best utilise available processing techniques to optimise sensor response through geometry and conversion media configurations, a reliable model is required. This must assess the performance in terms of spectral response and overall efficiency as a function of detector and converter geometry. The same is also required for proper interpretation of experimental data. Sensors have been fabricated with varying metallisation schemes indented to permit high temperature operation; Present test results indicate that viable fabrication schemes for high temperature contacts have been developed and present modelling results, supported by preliminary data from partners indicate simulations provide a useful representation of response.
\end{abstract}

Keywords: Diamond, High temperature, Dosimetry, Radiation sensor

\section{Introduction}

This body of work aims to develop spectroscopic radiation sensors for applications where high temperature operation and radiation hardness are required, with a particular interest in detection performance for neutrons and gamma-rays. Our work has focussed on the development of diamond detectors with suitable metallisation that will survive the required operating temperatures $\left(>200^{\circ} \mathrm{C}\right.$ ) without degradation. We have modelled the detector response, with a particular interest in the use of conversion media to improve thermal neutron detection, and possible gains in efficiency and position sensitivity that could be achieved by going beyond simple planar geometries (made possible by plasma processing of the diamond substrates and application of appropriate contact layouts).

Recent progress in the use of plasma processing to deliver complex surface geometries has yielded results that suggest the technique is a viable method of enhancing detector response, such use having been reported in the literature on a coarse scale for thinning[1] and surface processing and patterning[2]. To make the best use of this capability it is vital that the relationship between the diamond surface/conversion media interface geometry and detector performance is well understood (it is obvious that increasing the angular coverage of a converter by the detector will improve response, but it must be well quantified) in order to make efficient guided development choices for real

\footnotetext{
${ }^{*}$ Corresponding author

Email address: Alex.Metcalfe@brunel.ac.uk (Alex Metcalfe)
}

devices with a material that is still very expensive and challenging to process.

To achieve this, the physics of the interactions between incident radiation and the detector system must be adequately simulated. Due to the number of parameters and the probabilistic nature of the different radiation interactions involved, simple models very quickly give non-physical outcomes on this type of system so a more complex approach, making use of the large amounts of calculating power readily afforded by modern computers must be used. To accomplish this models have been constructed in the fully integrated radiation transport packages FLUKA2011[3, 4] and MCNPv6[5]

\section{High Temperature Sensors}

\subsection{Fabrication of High Temperature Diamond Sensors}

Sensors have been fabricated using vacuum metallisation facilities at Brunel University London and commercially available single crystal diamond substrates marketed by the supplier as electronic grade. The metals applied were selected based on work carried out to develop coatings for high temperature operation on lower grade diamond substrates. Work on achieving this goal elsewhere [6] indicates that certain metals such as $\mathrm{Pt}$ are unsuitable for this application and as such, metals that will give good ohmic contact and adhesion have been explored, the best of which are presented here. 


\subsection{Testing Methodology}

Tests were carried out in small vacuum chamber (shown schematically in Figure 1) evacuated by a turbopump equiped dry pumping setup to a pressure $<5 \times 10^{-4}$ mbar. Heating of the sensors, each mounted to a test PCB, was accomplished using a copper block with an attached AIN heater element and platinum resistance thermometer within the chamber. This was attached by multi-way vacuum feedthrough to an external temperature controller (Lakeshore 331). Electronic readout of the system was carried out by use of vacuum feedthrough connectors to a standard preamp (Canberra model 2004, typically used for silicon devices) then NIM crate mounted shaping amplifier (Canberra model 2021) connected to a PC based multichannel analyser (Canberra Eagle Plus). To assess alpha particle spectroscopy performance the sensors were exposed to a small triple alpha source $\left({ }^{241} \mathrm{Am},{ }^{239} \mathrm{Pu},{ }^{244} \mathrm{Cm}\right.$ of $1 \mathrm{kBq}$ activity each).

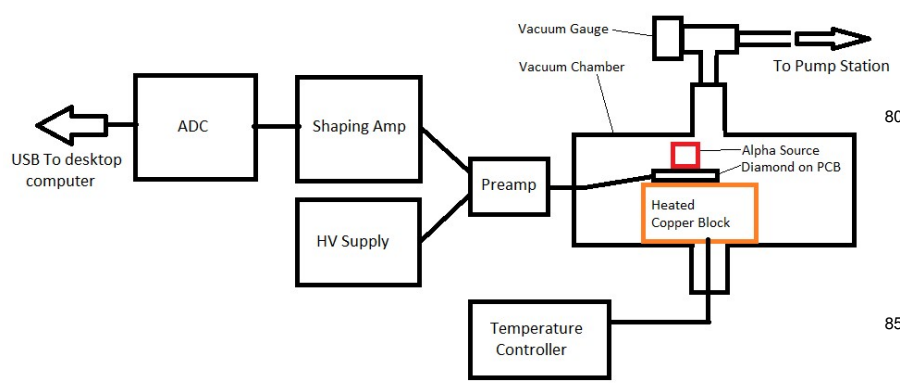

Figure 1: Schematic of setup for tests under alpha source irradiation.

\subsection{Testing Results}

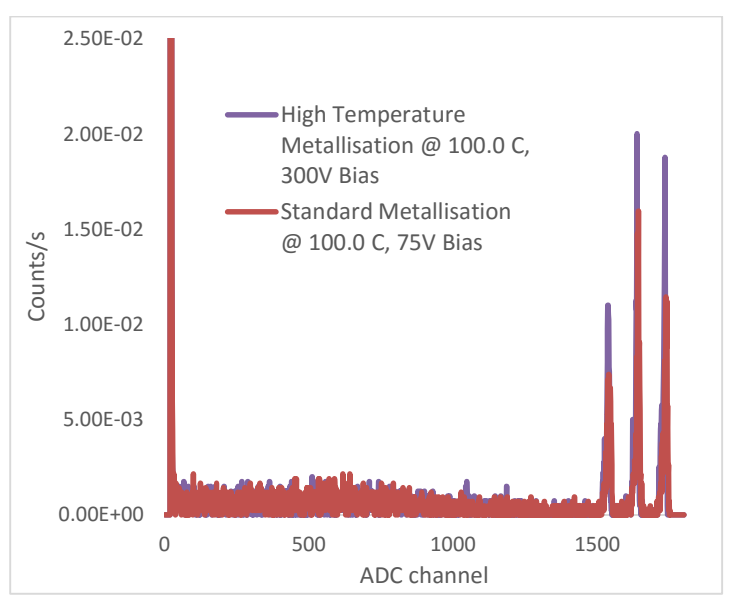

Figure 2: Spectra from triple alpha source for detectors with 'standard' and ${ }^{105}$ 'high temperature' coatings aquired at $100^{\circ} \mathrm{C}$.

\subsubsection{Discussion}

Initial tests at room temperature and $100{ }^{\circ} \mathrm{C}$ have yielded results that are indicative of a high quality detector for both $\mathrm{a}_{110}$ 70 'standard' metallisation and the high temperature compatible metallisation; both have retained the capability to clearly discern the peaks of the triple alpha source with very little shift in

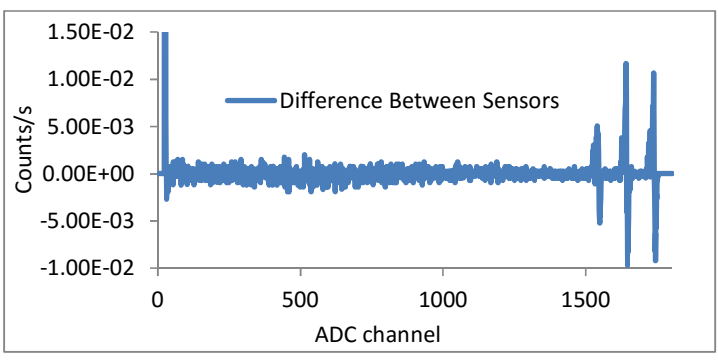

Figure 3: Residuals(high tempreture countrate - standard countrate) for Figure 2 .

peak position though with a notably larger peak magnitude for the high temperature metallisation, shown in Figure 2 with the difference clarified in Figure 3 Following this trend it is reasonable to expect that the detector will continue to function at temperatures above $100^{\circ} \mathrm{C}$, towards the target of at least $200^{\circ} \mathrm{C}[7]$ and this will be explored in further work. Also potentially noteworthy is that the detector with what is considered a standard metallisation was limited to lower bias operation to maintain full stability; increasing detector bias above $100 \mathrm{~V}$ resulted in the detector chain being overwhelmed with noise to circa 100 $\%$ dead time, recovery from which required the sensor to be left unbiased for a time on the order of hours. The leakage current was measured to be $0.63 \mathrm{pA}$ at $100 \mathrm{~V}$, compared with 0.014 $\mathrm{pA}$ for the high temperature metallisation. It is thought may be attributable to contamination of the subtrate surface prior to metallisation, and this discrepancy will be investigated fully in the immediate future.

\section{Modelling of Diamond Sensors}

\subsection{General Comments}

In order to extend the detection capabilities of a diamond sensor to thermal neutrons the addition of a conversion material to the basic detectors (presented in the previous results) 95 is required. models have been constructed to assess the contributions to the detector signal, and how they are affected by geometry, for varied sources of irradiation, principally thermal neutrons of energy around $2 \times 10^{-11} \mathrm{GeV}$. To this end a model has been set up to initially faithfully represent the geometry of se planar sandwich type sensor geometries. This was then extended to model the effects of a coating to convert thermal neutrons (boron, initially in $100 \%{ }^{10} \mathrm{~B}$ enriched form, then refined to also represent natural abundances). Finally the model was further modified to represent a detector for which the diamond surface is a set of square ridge trenches, which are filled with the conversion media.

\subsection{FLUKA2011}

FLUKA was the first package used in this work, and its capabilities to model radiation transport for most common scenarios is well suited to the evaluation of conversion media, however, it is subject to certain limitations. It treats transport of what it refers to as low energy neutrons, neutrons with energy $<20$ $\mathrm{MeV}$ with a system using energy groups. This does not readily 
permit the production and transport of correlated secondary particles, and instead deposits all energy from a nuclear interaction and its subsequent secondaries in an averaged fashion along the particle track, by use of calculated kerma factors. This leads to non representative spectra when attempting to fully simulate detector energy deposition, as this is reliant on accuracy of each event rather than on average. Above this threshold FLUKA uses models, with fully point wise transport. This permits simulation of such effects, assuming the model used matches data tables accurately in the correct channels.

\section{3. $M C N P v 6$}

Due to the limitations in FLUKA and also the general need for cross checking, the models set up in FLUKA were repeated in MCNPv6. MCNPv6 uses a continuous cross section table rather than grouped energy approach to neutron transport and as such is not implicitly limited in the $<20 \mathrm{MeV}$ range as FLUKA is. It is however still somewhat limited in the regime for simulating direct detection interactions in carbon due to the data tables available and the models used in the absence of such.

\subsection{Modelling Results}

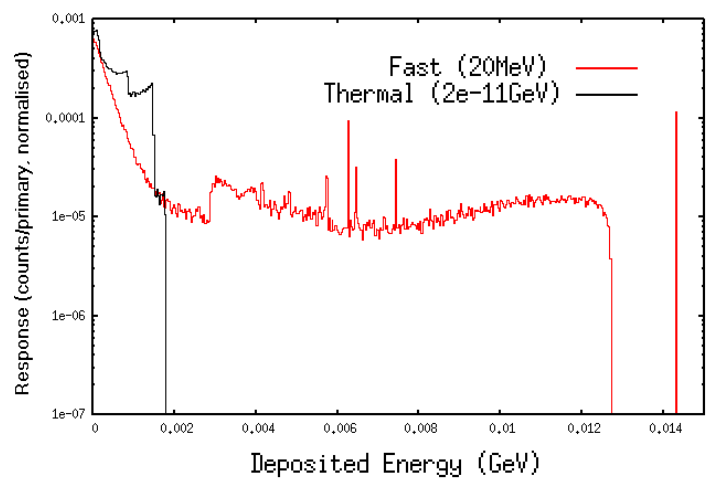

Figure 4: Simulated response of a converter coated diamond to neutrons, from FLUKA.

\subsubsection{Modelling Discussion}

From the results obtained from FLUKA and MCNPv6 it is possible to draw some key conclusions with regard to the optimisation of a detector consisting of a conversion layer and active diamond substrate. As Figure 4 shows there is a distinct separation between the features from the conversion of thermal neutrons and the direct response in the media from fast neutrons. Due to the short, well defined ranges of the heavy conversion reaction products there is a distinctly observed optimal thickness for planar conversion media of $7 \%$ at $\approx 3 \mu \mathrm{m}$, for the simulated conditions as shown in Figure 5. Due to contributions from the relatively highly penetrating gamma emission of boron under thermal neutrons there is an additional, though relatively small factor, that is dependent on the thickness of the substrate, a dimension that does not affect the alpha and ion detection until thicknesses on par with their penetration ranges

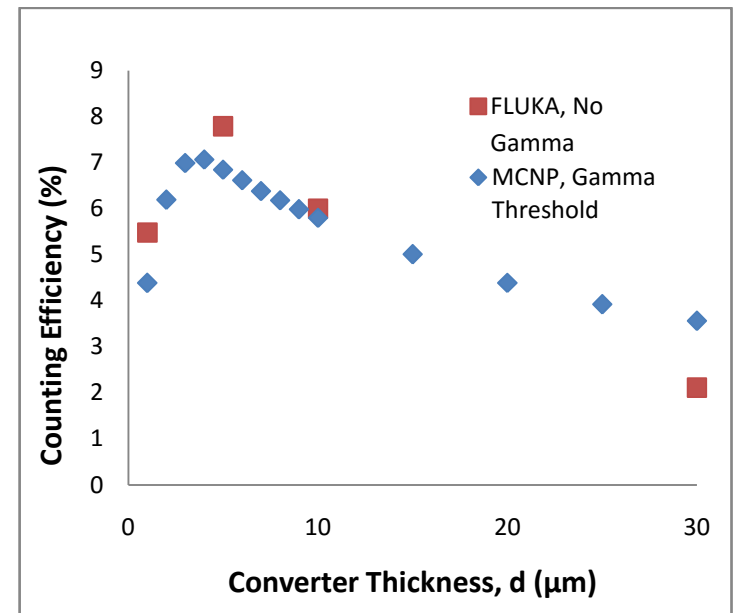

Figure 5: Counting efficiencies for thermal neutrons,with varied converter thickness.

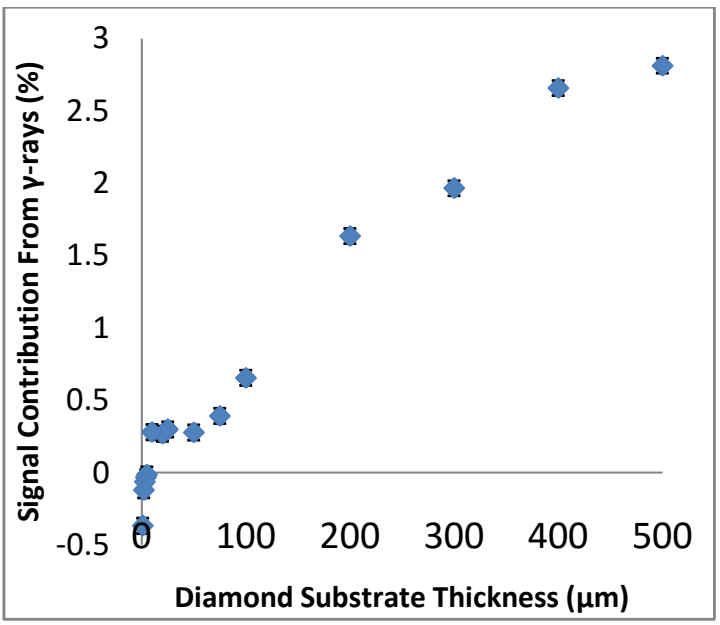

Figure 6: Simulation of contribution of gamma rays to detector signal with respect to thickness of the detector substrate, for a converter that produces both alpha and gamma radiation, from FLUKA.

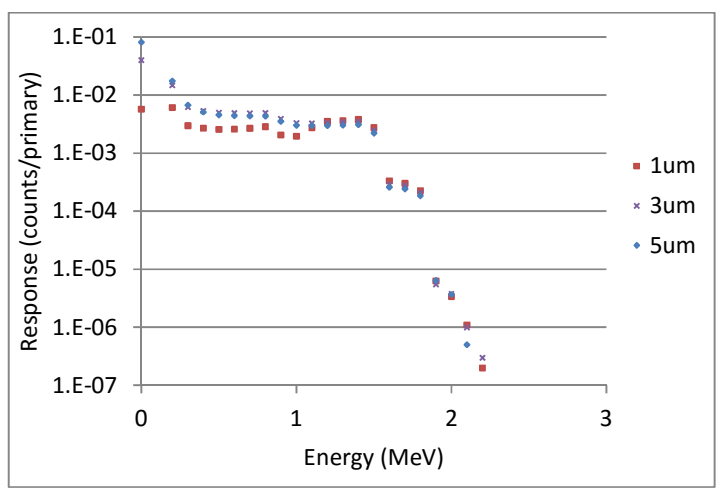

Figure 7: Simulated spectra with variation of converter thickness for planer detector, from FLUKA. 


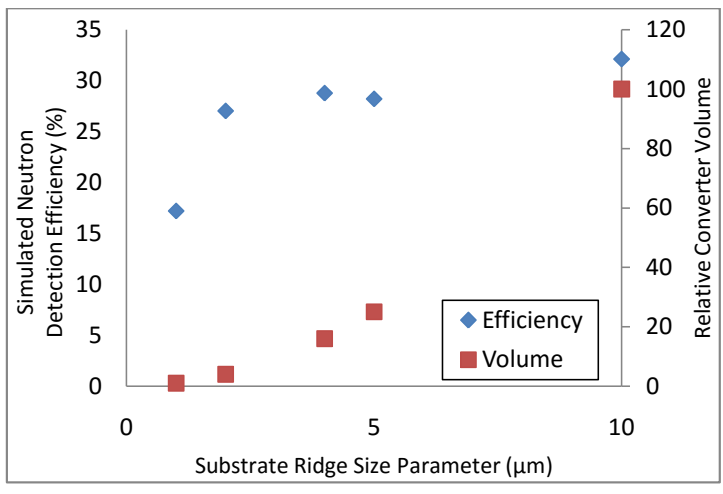

Figure 8: Simulated effect of a substrate/converter interface with square ridges, of varied size on the detection efficiency of thermal neutrons, from FLUKA.

are reached, shown by the negative gamma contribution artefact in Figure 6 for very thin $<5 \mu \mathrm{m}$ substrates. The difference in detection contribution between gamma, alpha and ion contributions is also starkly illustrated in Figure 7 , increasing linearly at ${ }^{205}$ the very low energy end of the spectra, whereas self shielding is already notable at $5 \mu \mathrm{m}$ of thickness.

The results for a $3 \mathrm{~d}$ converter/diamond surface show a distinct diminishing return as the size of the converter inclusions is increased, well below scaling with the relative filled area, as Figure 8 demonstrates. These $3 \mathrm{~d}$ surface results indicate that a210 patterned surface detector may be a viable way to increase performance, without resorting to use of structures with multiple crystals sandwiching conversion media[8, 9]. In terms of spectral response, it has been shown that the response from thermal 215 neutrons interacting with the conversion media results in a refrom secondary particles that is dependent mainly on the thickness and composition of the applied conversion media, such that determination of the presence of low energy neutrons220 may be well quantifiable.

Attempts to model neutrons at $14 \mathrm{MeV}$ have been less sucul, with the identification of weaknesses in FLUKA in this energy range, though results at thermal energies and from av-225 eraged energy deposition suggest that the models used here are at least an approximate representation. Use of MCNPv6 has supported these results from FLUKA in most circum-

175 stances, though differing somewhat radically in this energy ${ }^{230}$ range, though paths to achieve a model of response in MCNP have been identified, involving either some post-processing of data generated by carefully setup tallies, to multiply fluxes by cross sections in a simple manner[10], or the use of more in-235 depth methods involving user routines to permit reconstruction via forcing the generation of secondary particles[11].

\section{Conclusions}

Monte Carlo modelling of diamond detectors has been found to agree with findings as presented in literature and observed experimental results, with further validation impending from tests against more complex experimental setups, though refinements in the above thermal, $<20 \mathrm{MeV}$ range are required to compensate for the deficiencies in data tables available. The work carried out also indicates that a $3 \mathrm{~d}$ converter-substrate interface is a viable way to increase thermal neutron detection efficiency. The simulations show 4.2 times improvement. This work suggests that the high temperature coatings tested so far are viable mechanically, and preliminary alpha spectroscopy tests at elevated temperatures suggest that the fabricated detectors suffer very minimal degradation in quality at $100^{\circ} \mathrm{C}$ and will likely perform well at higher temperatures, which will be explored in further work.

\section{Acknowledgements}

For funding the academic and industrial efforts (respectively) on this project The authors acknowledge the EPSRC (Engineering and Physical Sciences Research Council) Grant: EP/L504671/1 and InnovateUK. The authors also wish to acknowledge the industrial partners involved in, Micron Semiconductor Limited and Schlumberger Limited for their roles in development and testing as part of this collaborative project.

\section{References}

[1] M. Pomorski, B. Caylar, P. Bergonzo, Super-thin single crystal diamond membrane radiation detectors, Applied Physics Letters 103 (11).

[2] D. T. Tran, C. Fansler, T. A. Grotjohn, D. K. Reinhard, J. Asmussen, Investigation of mask selectivities and diamond etching using microwave plasma-assisted etching, Diamond \& Related Materials 19 (7) (2010) 778-782.

[3] T. Bohlen, F. Cerutti, M. Chin, A. Fosso, A. Ferrari, P. Ortega, A. Mairani, P. Sala, G. Smirnov, V. Vlachoudisl, The FLUKA code: Developments and challenges for high energy and medical applications, NUCLEAR DATA SHEETS 120 (2014) 211-214.

[4] A. Ferrari, P. Sala, A. Fasso, J. Ranft, FLUKA: a multi-particle transport code cern-2005-10, CERN200510 (2005), INFN/TC_0511, SLACR773.

[5] T. Goorley, M. James, T. Booth, F. Brown, J. Bull, L. J. Cox, J. Durkee, J. Elson, M. Fensin, R. A. Forster, J. Hendricks, H. G. Hughes, R. Johns, B. Kiedrowski, R. Martz, S. Mashnik, G. McKinney, D. Pelowitz, R. Prael, J. Sweezy, L. Waters, T. Wilcox, T. Zukaitis, Initial MCNP6 release overview, Nuclear Technology 180 (3) (2012) 298-315.

[6] M. Angelone, N. Fonnesu, M. Pillon, G. Prestopino, F. Sarto, E. Milani, M. Marinelli, C. Verona, G. Verona-Rinati, Spectrometric performances of monocrystalline artificial diamond detectors operated at high temperature, IEEE Transactions on Nuclear Science 59 (5) (2012) 2416-2423.

[7] R. Pilotti, M. Angelone, S. Loreti, G. Pagano, M. Pillon, F. Sarto, M. Marinelli, E. Milani, G. Prestopino, C. Verona, G. Verona-Rinati, High temperature performances of cvd single crystal diamond detectors, Proceedings of Science 2015.

[8] A. Pietropaolo, G. Verona Rinati, C. Verona, E. M. Schooneveld, M. Angelone, M. Pillon, A single-crystal diamond-based thermal neutron beam monitor for instruments at pulsed neutron sources, Nuclear Inst. and Methods in Physics Research, A 610 (3) (2009) 677-681.

[9] D. S. McGregor, R. T. Klann, H. K. Gersch, J. D. Sanders, Designs for thin-film-coated semiconductor thermal neutron detectors, in: Nuclear Science Symposium Conference Record (2001 IEEE), Vol. 4, 2001, pp. 2454-2458

10] E. Lukosi, M. Prelas, J. Palsmeier, Monte Carlo simulations of multiplexed electronic grade CVD diamond for neutron detection, Radiation Measurements 47 (6) (2012) 417-425.

[11] A. Milocco, M. Pillon, M. Angelone, A. Plompen, A. Krsa, A. Trkov, Monte Carlo simulation of the experimental pulse height spectra produced in diamond detectors by quasi-mono-energetic neutrons, Nuclear Inst. and Methods in Physics Research, A 720 (2013) 74-77. 